\title{
BOTÁNICA EN LAS MISIONES JESUÍTICAS DE AMÉRICA ESPAÑOLA Y EL PACÍFICO Y LAS CONTRIBUCIONES DE JESUITAS CENTROEUROPEOS, SIGLOS XVII Y XVIII ${ }^{1}$
}

\author{
por MARKÉTA KŘÍŽOVÁ \\ (Universidad Carolina)
}

\begin{abstract}
The present text focuses on the problem of European intellectual history and history of science, in relation to the conquest and colonization of overseas regions, especially America. The various problems of intellectual development and cultural transfers, brought about by the general opening of new horizonts, are discussed on the basis of documentation produced by the missionaries from the Central European provinces of the Society of Jesus, active in the New World in the 17th and 18th centuries. The text summarizes the opinions and stereotypes about the American flora formulated by the Jesuits, as well as their efforts to understand not only the laws of nature, but laws of universe through its study; and, at the same time, hints at the specific features of the Central European gaze upon the New World.
\end{abstract}

Keywords: colonization; America; Society of Jesus; missions; botany; history of science; cultural transfer; Central Europe; 17th-18th century

El descubrimiento y colonización de las regiones ultramarinas ciertamente abrieron para los europeos un "nuevo mundo", lleno de fenómenos que instaban a la exploración y clasificación y a la subsecuente revisión de los conocimientos intelectuales de su tiempo. Entre las disciplinas que habían sido estimuladas hasta el extremo por las conquistas ultramarinas, la botánica ocupó un puesto prominente. En cierto respecto, esta disciplina encarna el vínculo entre el desarrollo científico y la expansión imperial. Al lado de la mera curiosidad intelectual, los esfuerzos por aprovecharse económicamente de las regiones nuevamente conquistadas, por buscar los productos vendibles y procesables, por no solamente conocer, sino reconfigurar la naturaleza, marcaban la exploración del ultramar desde las navegaciones de los portugueses a lo largo de África y el primer viaje de Colón. ${ }^{2}$

1 La primera versión de este texto se presentó durante el simposio "Mutis, Haenke. Ciencias naturales europeas y América del siglo XVIII" que tuvo lugar el 14 de noviembre 2016 en el Instituto Cervantes de Praga, con ocasión de la donación de la edición de la obra de José Celestino Mutis por parte de la Embajada de España en Praga a la Biblioteca del Centro de Estudios Ibero-Americanos de la Universidad Carolina. La presentación se reelaboró y completó en la Universidad Carolina dentro del proyecto de la fundación europea para el desarrollo regional "Creativity and Adaptability as Conditions of the Success of Europe in an Interrelated World" (No. CZ.02.1.01/0.0/0.0/16_019/0000734).

2 En el diario de a bordo de Colón aparecen repetidamente las alusiones a plantas y árboles "de grande provecho". De las muchas ediciones modernas de esta fuente, usé Diario del primer y tercer viaje de Cristóbal Colón, ed. Consuelo Varela (Obras Completas de Bartolomé de Las Casas, vol. 14), Madrid 
Aunque ciertamente no se puede reducir el desarrollo científico en las vísperas de la modernidad al mero apéndice del esquema imperial, lo que Paula de Vos llamó "botánica económica"3, sin duda constituía un importante componente de la naciente disciplina. Los intereses intelectuales conformaban a los cambios de los mercados provocando estos cambios y a la vez respondían a los planes de expansión colonial. ${ }^{4}$ A la vez, sin embargo, reflejaban en el estudio de la botánica y otras disciplinas del estudio de la naturaleza las corrientes dinámicas de la filosofía europeas, el humanismo, la Reforma Católica, tanto como la temprana Ilustración. Todo esto se puede ver claramente en el caso de los estudios botánicos realizados en el suelo americano por los miembros de la Compañía de Jesús, que constituyen el tema principal del presente trabajo.

\section{La ciencia jesuita}

A través de tres siglos de su existencia, la Compañía de Jesús participaba activamente en el intercambio de conocimientos entre el Viejo y el Nuevo Mundo. La historiografía moderna ha, desde hace unos decenios, rebatido la imagen de los jesuitas de retrógrados bigotes que se oponían a conocimientos y enfoques empíricos, tanto

1989, aquí pp. 69 (martes, 23 de octubre), 77 (4 de noviembre), etc. Del temprano interés español en la botánica americana se puede mencionar, por ejemplo, la expedición farmacéutico-botánica a México, realizada en la segunda mitad del siglo XVI por el "protomédico real" Francisco Hernández, que produjo 15 volúmenes de dibujos y textos (parte de ellos perdida en el incendio del Escorial en el siglo XVII). En todo, Hernández identificó tres mil plantas mexicanas. Simon VAREY et al., The Mexican Treasury: The Writings of Dr. Francisco Hernández, trad. Rafael Chabrín, Cynthia L. Chamberlein, Simon Varey, Stanford 2001; José María LÓPEZ PIÑERO - José PARDO TOMÁS, La influencia de Francisco Hernández. (1515-1587) en la constitución de la botánica y la materia médica moderna, Valencia 1994. En el mismo período originó el Libellus de medicinalibus Indorum herbis, compuesto por dos educados nahuas, Juan Badiano y Martín de la Cruz. The Badianus Manuscript, trad. Emily Walcott Emmart, Baltimore 1940; ambos proyectos mencionados en Jorge CANIZARES-ESGUERRA, Nature, Empire and Nation: Explorations of the History of Science in the Iberian World, Stanford 2006, pp. 7-8.

3 Paula DE VOS, "The Science of Spices: Empiricism and Economic Botany in the Early Spanish Empire", Journal of World History 17/4, Honolulu 2006, pp. 399-427.

4 Además del texto ya citado de Paula de Vos, véase su otro artículo, Paula DE VOS, “An Herbal El Dorado: The Quest for Botanical Wealth in the Spanish Empire", Endeavour 27, 2003, pp. 117-121, accesible de: http://www.sciencedirect.com/science/article/pii/S0160932703001091, [consultado el 2 de enero de 2020]; en la misma línea, Londa SCHIEBINGER, Plants and Empire: Colonial Bioprospecting in the Atlantic World, Cambridge, MA. 2004; Londa SCHJIEBINGER - Claudia SWAN (eds.), Colonial botany: science, commerce and politics in the early modern world, Philadelphia 2004; Sarah EASTERBY-SMITH, Cultivating Commerce: Cultures of Botany in Britain and France, 1760-1815, Cambridge 2017; Lucile H. BROCKWAY, "Science and Colonial expansion: The role of the British Royal Botanic Gardens", American Ethnologist 6/3, Arlington, VA 1979 , pp. 449-465; Zaheer BABER, "The Plants of Empire: Botanic Gardens, Colonial Power and Botanical Knowledge”, Journal of Contemporary Asia 46:4 (2016), pp. 659-679. Incluso la clasificación de Carlos Linneo recientemente ha sido integrada en este marco interpretativo, ya que -según los historiadores- salía de y hubiera sido usada por los esfuerzos expansionistas, de matiz marcadamente nacional. Lisbet KOERNER, Linnaeus: Nature and Nation, Cambridge, MA 1999. 
como el general cliché del conflicto entre la religión y la ciencia que supuestamente precedía a la victoria final de la Ilustración. ${ }^{5}$ Por supuesto, "ciencia" es una palabra problemática, y algunos historiadores dudan de su uso para el tiempo Temprano Moderno. ${ }^{6}$ Pero está claro hoy en día que la mentalidad barroca, que combinaba la fe en intervenciones divinas con experimentación, ciertamente no presentaba el impedimento a la formulación de modelos de conocimiento rigurosos.

Aunque hubo también otras órdenes que alcanzaron logros culturales y científicos, las actividades científicas practicadas por los jesuitas se distinguían por su longevidad y multidisciplinaridad, y también por lo que Steven Harris denominó "cultura corporativa", en la que los nexos intelectuales, los colegios y universidades jesuitas, que servían para procesamiento y diseminación de los conocimientos habían sido aprovisionados por material de varias partes del mundo, de Europa, América, África y Asia, por los miembros de la Orden que servían en estas partes.? La circulación de hombres, textos y objetos dentro de la Orden, entre sus centros administrativos y educacionales en Europa y las misiones en el ultramar instó la producción de un cuerpo de conocimientos impresionante. Además, obraba el cuidado jesuita en preservar y organizar su documentación lo que hacía posible a los miembros de la Compañía de Jesús familiarizarse con y desarrollar los hallazgos de sus predecesores. ${ }^{8}$

Ignacio de Loyola envisionó la Compañía de Jesús como una agrupación principalmente misional y predicadora, con el objetivo de asistir en la reforma general de la comunidad cristiana. Pero la búsqueda de miembros preparados espiritual

5 Daniela BLEICHMAR et al. (eds.), Science in the Spanish and Portuguese Empires, 1500-1800, Stanford 2009; Antonio BARRERA-OSORIO, Experiencing Nature: The Spanish American Empire and the Early Scientific Revolution, Austin 2006.

6 La justificación del término, a pesar de estas dudas, en Jorge CAÑIZARES-ESGUERRA, Nature, Empire and Nation: Explorations of the History of Science in the Iberian World, Stanford 2006, p. 3.

7 Steven J. HARRIS, "Confession-Building, Long-Distance Networks, and the Organization of Jesuit Science", Early Science and Medicine 1/3, Leiden/Boston 1996, pp. 287-318; el mismo, "Mapping Jesuit Science: The Role of Travel in the Geography of Knowledge", in: John O'Malley et al. (eds.), Jesuits: Cultures, Sciences, and the Arts 1540-1773, vol. 1, Toronto 1999, pp. 212-240; Steven J. HARRIS, "Jesuit Scientific Activity in the Overseas Missions, 1540-1773", Isis 96/1, Chicago 2005, pp. 71-79.

8 Para los archivos jesuitas véase Marcus FRIDRICH, "Archives as networks: The geography of record-keeping in the Society of Jesus (1540-1773)", Archival Science 10/3, Blacksburg 2010, pp. 285298; para la circulación de la información, Luis MILLONES FIGUEROA, "La intelligentsia jesuita y la naturaleza del Nuevo Mundo en el siglo XVII", in: Domingo Ledezma - Luis Millones FIGUEROA (eds.), El saber de los jesuitas, historias naturales y el Nuevo Mundo, Frankfurt a. M./Madrid, 2005, pp. 27-51. En lo que toca la ciencia jesuita en general, se pueden citar algunos textos in: John O'MALLEY et al. (eds.), Jesuits: Cultures, Sciences, and the Arts 1540-1773, Toronto 1999, 2005, 2 vols.; Mordechai FEINGOLD (ed.), Jesuit Science and the Republic of Letters, Cambridge 2003 ambos libros colectivos centran más bien a Europa que a las misiones; y también Agustín UDÍAS, Jesuit Contribution to Science: A History, Cham 2015; Andrés I. PRIETO, Missionary Scientists: Jesuit Science in Spanish South America, 1570-1810, Nashville 2011; Miguel de ASÚA, Science in the Vanished Arcadia: Knowledge of Nature in the Jesuit Missions of Paraguay and Río de la Plata, Leiden/Boston 2014; Mark WADDELL, Jesuit Science and the End of Nature's Secrets, Farnham 2015 - estas obras mencionan extensamente la ciencia en el contexto de las misiones jesuitas ultramarinas. 
y académicamente para la obra misional llevó a los líderes a centrarse en la enseñanza, lo que se convirtió rápidamente en una de sus tareas más importantes. Ya en 1560 Juan de Polanco escribió a todos los superiores jesuitas que precisamente la educación de la juventud fuera en adelante el ministerio de la Orden. ${ }^{9}$ Pronto, los jesuitas controlaron una red extensa de escuelas de todos los niveles, unas 800 instituciones en Europa, América Latina, África y Asia. ${ }^{10}$

A través de sus institutos educacionales, podían comunicarse con los otros sabios de su época; y dentro de la Orden discutían e intercambiaban experiencias miembros de muchas naciones, graduados de varias universidades.$^{11}$ La enseñanza cubría no solamente la teología y las humanidades, sino también rudimentos de filosofía, matemática y también de historia natural. Al lado de los colegios jesuitas se fundaban observatorios astronómicos y jardines botánicos. El estudio de la naturaleza era generalmente aceptado en el período bajo consideración como una actividad intelectual que permitía acercarse al Creador. La metáfora de la naturaleza como un "libro" complementario a la Biblia era popular en las obras de sabios renacentistas y barrocos en general, y también entre los autores de la Compañía. ${ }^{12}$ Más aún, los jesuitas llegaron a entender el estudio, como tal, a manera de un empleo estrechamente relacionado con la piedad y la vida especial, de hecho equivalente al rezo y a la contemplación. ${ }^{13}$

Es verdad que a veces el sistema escolar jesuita era criticado como "estéril" ya por sus contemporáneos del siglo XVII. ${ }^{14}$ Pero en general, la Compañía de Jesús, a pesar de ser una corporación religiosa que consistía mayoritariamente en sacerdotes ordenados, comprometidos al cuidado de las almas, en comparación con otras órdenes de la Edad Temprana Moderna, obraba con una orientación inequívoca al mundo y sus necesidades. La mayoría de los jesuitas pasaban su tiempo en el espacio seglar, no en el púlpito o en el monasterio; y también en su trabajo intelectual ellos se interesaban en las ciencias prácticas y en el manejo de asuntos mundanos. ${ }^{15}$

La multitud de novedades y problemas encontrados en el ultramar que la autoridad de filósofos y teólogos antiguos no había podido satisfacer motivó el cuestionamiento del pensamiento escolástico de parte de algunos miembros de la Compañía. Esto testifica una de las más populares crónicas jesuitas de este tiempo, la Historia natural y moral de las Indias de José de Acosta. En el prefacio Acosta notó que mientras que otros autores habían escrito sobre América, "hasta ahora no he visto

9 John O’MALLEY, The First Jesuits, Cambridge, MA. 1995, p. 200.

10 PRIETO, Missionary Scientists, p. 94.

11 Víctor NAVARRO, "Tradition and Scientific Change in Early Modern Spain: The Role of the Jesuits", in: Feingold (ed.), Jesuit Science, pp. 331-332.

12 MILLONES FIGUEROA, "La intelligentsia jesuita", p. 36.

13 PRIETO, Missionary Scientists, p. 3.

14 Mikes Kelemen, el paje del exiliado Francisco II Rákóczi, en su carta de 1727 se quejó que los estudiantes en las escuelas jesuitas pasaban los primeros ocho años aprendiendo latín y nada más, "sin provecho alguno para ellos o para su país". (Cit. por Domokos KOSÁRY, Culture and Society in Eighteenth-Century Hungary, trans. Zsuzsa Béres, Budapest 1987, p. 108.)

15 John O'MALLEY, "Introduction”, in: idem et al. (eds.), vol. 2, p. xxxiii. 
autor que trate de declarar las causas y razon de tales novedades y extrañezas de naturaleza, ni que haga discurso e inquisicion en esta parte; ni tampoco he encontrado libro, cuyo argumento sea los hechos é historia de los mismos Indios antiguos y naturales habitadores del nuevo orbe". ${ }^{16}$ Se verá más abajo en el presente artículo que precisamente las consultaciones con los indígenas facilitaron a los jesuitas sus grandes avances (no solamente) en el área de la botánica. Regresando a Acosta, éste al discutir los problemas del clima tropical incluso abiertamente puso en duda las opiniones de Aristóteles. "Aunque tan gran Filósofo, se engañó en esta parte." ${ }^{17} \mathrm{Su}$ proclamación de la superioridad de la observación empírica sobre la autoridad escolástica reflejó lo que ya se notó acerca de la "ciencia jesuita" en general - confianza en la observación y experimento, a expensas de la fe en las autoridades, y un anhelo por penetrar en las mismísimas causas de cosas.

En total, entre 1540 y 1773 los miembros de la Compañía de Jesús produjeron un corpus de más de 5000 libros, panfletos y breves textos refiriéndose a varias ramas de las ciencias naturales existentes en el tiempo; ${ }^{18}$ y muchos de éstos reflejaban las realidades del Nuevo Mundo. Por cierto, como constaron los dos editores del valioso volumen dedicado precisamente a la ciencia jesuita y el Nuevo Mundo, los jesuitas atravesaron el Atlántico principalmente para convertir y educar. ${ }^{19} \mathrm{La}$ comprensión de las sociedades indígenas y la naturaleza que los rodeaba no constituía el objetivo per se, solamente contribuía a realizar sus propósitos, tanto como la propagación de sus trabajos en este respecto elevaba el prestigio de la Orden y atraía a los ricos patrones. Ya Jerónimo Nadal, uno de los fundadores de la Compañía de Jesús, comparó los ejercicios intelectuales al "anzuelo, con el que nosotros pescamos las almas" ${ }^{20} \mathrm{Al}$ fin y al cabo, el esplendor natural del Nuevo Mundo en los ojos de los jesuitas solamente comprobó la existencia de un plano divino de profundidad incomprensible.

El mismo Acosta concluyó el prefacio arriba citado de Historia, con palabras que no ponen en duda la tarea principal de la Compañía de Jesús. "El fin de este trabajo es, que por la noticia de las obras naturales el que Autor tan sabio de toda naturaleza ha hecho, se le dé alabanza y gloria al altísimo Dios, que es maravilloso en todas partes. Y por el conocimiento de las costumbres y cosas propias de los Indios, ellos sean ayudados á conseguir y permanecer en la gracia de la alta vocacion del

16 José de ACOSTA, Historia natural y moral de las Indias [1590], Madrid 1894, pp. xiii-xiv. (La primera versión latina se publicó bajo el título De natura Novi Orbis en 1588; la más voluminosa Historia natural y moral de las Indias, cuyos primeros capítulos son su propia versión traducida al castellano de De natura Novi Orbis, salió a la luz en 1590. Se publicaron cuatro ediciones españolas de esta obra en menos de veinte años, y hasta los principios del siglo XVII también traducciones al latín, italiano, francés, alemán e inglés.) Véase Fermín del PINO, "La Historia natural y moral de las Indias como género: orden y génesis literaria de la obra de Acosta”, Histórica 24:2 (2000), pp. 295-326.

17 ACOSTA, Historia natural y moral, p. 40.

18 HARRIS, "Confession-Building, Long-Distance Networks", p. 288.

19 Domingo LEDEZMA - Luis MILLONES FIGUEROA, "Introducción: Los jesuitas y el conocimiento de la naturaleza americana”, in: idem (eds.), El saber de los jesuitas, historias naturales y el Nuevo Mundo, Frankfurt a. M./Madrid, 2005, p. 9.

20 Jerónimo NADAL, "Exhortatio Coloniensis 6a" (1567), cit. por WADDELL, Jesuit Science, p. 4. 
Santo Evangelio." ${ }^{21}$ Los miembros de la Compañía de Jesús tenían que conformarse con las reglas que determinaban su vida cotidiana y su horizonte intelectual, y sus textos se sujetaban a la censura interna..$^{22}$ Pero dada su preparación intelectual, los misioneros e intelectuales jesuitas fueron intérpretes privilegiados de la naturaleza americana. Sus textos alcanzaban no solamente lectores católicos, sino también los cultos protestantes. ${ }^{23}$ Entre otros temas, esto vale también para sus estudios botánicos, realizados en el ultramar, que constituyen el tema principal del presente texto.

No es posible ni siquiera enumerar en este espacio limitado los textos producidos por los misioneros jesuitas en las posesiones españolas en América y en las islas pacíficas, en torno a la flora local ${ }^{24}$ Por esto, la atención se prestará en especial aunque no exclusivamente- a las contribuciones de los jesuitas de Europa central. ${ }^{25}$ Gracias a su participación en el proyecto misional, estos miembros de la Compañía de Jesús intermediaban los conocimientos científicos con sus socios y el público general en sus provincias de origen, para quienes las informaciones del ultramar mantenían un gusto eminentemente exótico y difícilmente accesible. Así, ampliaron más allá la red transnacional de informaciones y testimonios que dio origen a la ciencia moderna.

21 ACOSTA, Historia, pp. xiv-xv.

22 Para la censura dentro de la Orden, véase Marcus HELLYER, “Because the Authority of My Superiors Commands': Censorship, Physics and the German Jesuits", Early Science and Medicina 1/3, Leiden/Boston 1996, pp. 319-354.

23 Por ejemplo, el célebre pensador protestante de origen moravo, Juan Amos Komenský (Comenio), leyó y citó a la Historia natural y moral del Nuevo Mundo del autor jesuita José de Acosta. Véase, por ejemplo, Joannes Amos COMENIUS, Novissima linguarum methodus (1648), in: Joannes Amos Comenius, Opera didactica omnia, Praga 1957, tomo. 1, p. 46. Galaxis Borja González asegura que también en Alemania los libros de los jesuitas rebasaban las fronteras confesionales y se encontraban en las bibliotecas probadas de los letrados protestantes, donde constituían la mayoría de los pocos libros de tema americano. Galaxis BORJA GONZÁLEZ, "Libros americanos, autores jesuitas y público alemán: la literatura jesuítica americana en el mercado de libros del siglo XVIII", in: Karl Kohut - Ma. Cristina Torales Pacheco (eds.), Desde los confines de los imperios ibéricos: Los jesuitas de habla alemana en las misiones americanas, Frankfurt a. M./Madrid 2007, pp. 663-696.

24 Y, por supuesto, también las observaciones botánicas realizadas por los misioneros que obraban en las posesiones portuguesas en Brasil y en Asia, o en Canadá francesa, merecerían un análisis detallado, pero el presente texto está limitado por la extensión. Para algunas citas bibliográficas acerca de los botánicos jesuitas en las posesiones portuguesas, véase Palmira FONTES DA COSTA - Henrique LEITÃO, "Portuguese Imperial Science 1450-1800: A Historiographical Overview”, in: Daniela Bleichmar et al. (ed.), Science in the Spanish and Portuguese Empires, 1500-1800, Stanford 2009, p. 43. También se tuvo que dejar de lado la obra original del jesuita polaco Michał Piotr BOYM, Flora sinensis, publicada en Viena en 1656. Edición facsimilar electrónica accesible de: http://eod .vkol.cz/ii14866/ (consultado el 2de enero de 2020), para Boym véase Linda L. BARNES, Needles, Herbs, Gods, and Ghosts: China healing and the West to 1848, Cambridge, MA. 2005, pp. 30-31. Agradezco a la prof. Olga Lomová por informarme de esta fuente.

25 En total, algunos centenares de misioneros de los países centroeuropeos servían en varias partes de América Española. Véase, por ejemplo, KOHUT - TORALES PACHECO (eds.), Desde los confines; Markéta KŘÍŽOVÁ, La ciudad ideal en el desierto: Proyectos misionales de la Compañía de Jesús y la Iglesia Morava en la América colonial, Praga 2004; Simona BINKOVÁ, Markéta KŘÍŽOVÁ et al., Ir más allá... (Fuentes bohemicales para el estudio comparativo de la expansión colonial española en la temprana Edad Moderna), Praga 2016. 


\section{Del asombro a la funcionalidad}

Los jesuitas eran, en general, inclinados a describir con detalle todo lo nuevo y maravilloso que veían en el ultramar. De hecho, las reglas de la Orden les instaban a hacerlo, ya que sus cartas servían externamente como una eficaz propaganda del trabajo jesuita y como un instrumento de instrucción dentro de la Compañía. En una carta de 1554, Loyola instruyó a Gaspar Berce, misionero en India:

"Algunas personas principales, que en esta Ciudad [= Roma] leen con mucha edificación suya las letras de las Indias, suelen desear, y o piden diversas veces, que se escriviese algo de la cosmografía de las regiones donde andan los nuestros, como sería cuán luengos son los días de verano y de invierno, cuándo comienza el verano, si las sombras van sinistras, o a la mano diestra. Finalmente, si otras cosas hay que parezcan extraordinarias, se dé aviso, como de animales y plantas no conocidas, o no en tal grandeza, etc."26

En respuesta a tales exhortaciones, la correspondencia de los misioneros jesuitas puede servir hasta hoy día como una fuente importante para conocer la naturaleza ultramarina, tanto como las costumbres de sus habitantes. Además, influía la imaginación de la época. Las cartas circulaban en copias manuscritas, se leían en voz alta en refectorios y aulas de los colegios jesuitas o incluso se imprimían en traducciones a lenguas vernaculares, para ganar el más amplio público. ${ }^{27}$

En especial en los primeros días y semanas en el Nuevo Mundo los misioneros se maravillaban ante la inmensidad de las tierras americanas, la exuberancia de su flora y la fauna, la grandeza de las montañas. No es sorprendente que precisamente los miembros de la Orden oriundos de los países centroeuropeos se asombraran del ambiente del trópico. Se habían, por supuesto, aprovechado de algunos de los estereotipos tradicionales de la literatura más vieja -el topoi de la naturaleza desenfrenada en el primer lugar, ya que la Europa culta en este tiempo nuevamente descubrió el gusto para lo lejano, lo distinto, lo maravilloso- pero también notaban información factual muy concreta. Se pueden citar de las cartas casi al azar para encontrar alusiones a la flora americana. A menudo aparece el asombro de los misioneros por la ausencia de cereales en América y las descripciones del maíz y la mandioca, substituciones, en los ojos de los misioneros, más que insuficientes, para

26 Ignacio de Loyola a Gaspar Berce, Roma, 24-II-1554 (ARSI, Epp. 6,357-6,359), reproducido en Ignacio de LOYOLA, Obras (Edición manual), p. 853.

27 De antologías impresas de la correspondencia jesuita es, para la Europa Central, la más importante la editada entre los años 1726-1761 por Joseph Stöcklein citado con frecuencia como Weltbott o, sea, como Der Neue Welt-Bott [El nuevo mensajero del mundo]. Se trató de un compendium de cartas y resúmenes de cartas enviadas por misioneros jesuitas de varias partes del mundo, el continente americano entre ellos, pero también de China o África. Joseph STÖCKLEIN, Allerhand so Lehr-als Geist-reiche Brief-Schrifften und Reis-beschreibungen, welche von denen Missionarios der Gesellschaft Jesu aus Beyden Indien bis anno 1731 in Europa angelangt sind, Augsburg/Gratz/Wien 1726-1761, 37 tomos. Para los objetivos promocionales de la obra, véase Bernd HAUSBERGER, "El padre Joseph Stöcklein o el arte de inscribir el mundo a la fe", in: Kohut - Torales Pacheco (eds.), Desde los confines, pp. 631-659. 
el trigo y el centeno. ${ }^{28} \mathrm{Al}$ contrario, ellos degustaban con entusiasmo los frutos tropicales. El misionero de Bohemia Juan Tilpe describió en detalle el aguacate, la piña y en especial el plátano, su forma, color, consistencia y, por supuesto, el sabor del "fruto blanco, que parece a la mantequilla y literalmente se disuelve en la boca". ${ }^{29}$

Otro bohemio, Pablo Klein, quien se detuvo brevemente en el Caribe en viaje a su destinación final en las Filipinas, mencionó al cacao, "un tipo de nuez de Indias de que se hace una bebida llamada chocolate" y dedicó todo un párrafo al "milagro de la naturaleza", el coco, una fruta que brindaba "alimento, bebida, copino, aceite y medicamento". ${ }^{30}$ El alemán José Kropff durante la escala en la bahía de Ocoa en la isla Española admiró la fecundidad de la naturaleza caribeña: "La tierra es tan fértil que los árboles llevan todo el año o flores, o las frutas." ${ }^{31}$ Por supuesto no se trataba en estos casos de observaciones científicas propiamente dichas, descripciones rigurosas y menos aun análisis de fenómenos naturales. Con todo, las cartas enviadas a centroeuropa no carecían de importancia para el desarrollo intelectual en la región. Provocaron a los lectores -estudiantes en universidades jesuitas, o miembros de la Orden- contemplar y, quizás, cuestionar los tradicionales conceptos del mundo natural y su historia.

Al pasar a su destinación final, el asombro inicial dio lugar a los deberes cotidianos y los jesuitas centroeuropeos comenzaron a familiarizarse con sus entornos y aprovecharse de los recursos naturales del Nuevo Mundo. Aunque, como se expuso antes, su interés principal era el de llevar a los aborígenes al Cristo, y su interés en la naturaleza originalmente se basaba más bien en aficiones estéticas o espirituales -al admirar las bellezas de la naturaleza ellos alababan a Él que la creo-, en las misiones no podían sino centrar su atención en asuntos prácticos. Dados los escasos fondos para mantenerse a ellos mismos y a sus neófitos, se veían obligados a buscar recursos locales para alimentos, medicinas o incluso para su exportación. En breve, muy pronto comenzaron a esforzarse por explotar y "domar" la naturaleza indomable que ellos tanto habían admirado al principio.

28 Todas las cartas de los misioneros bohémicos sobre América, preservadas hasta hoy día en el suelo de la República Checa, fueron editadas por Pavel ZAVADIL, "Bohemia jesuitica in Indiis Occidentalibus. Latinská korespondence českých jezuitů z Ameriky, Filipín a Marián v českých a moravských archivech. Kritická edice" [Bohemia jesuitica in Indiis Occidentalibus. Correspondencia latina de los jesuitas bohemios de América, Filipinas y Marianas en los archivos checos y moravos], disertación doctoral, Universidad Carolina, Praga 2011 (mecanografiada), accesible de: https://is.cuni.cz/ webapps/zzp/detail/121385/, consulado el 2 de enero de 2020. A pesar de haber sido redactada la disertación en checo, las cartas se editaron y así se pueden consultar en su forma original, es decir, en latín. (La cita de la carta de Wenceslao Eymer, pp. 194-197, y de Pablo Klein, p. 400.)

29 Ibid., p. 598

30 ZAVADIL, "Bohemia jesuitica", pp. 399-400. Para el análisis más detallado de las primeras impresiones de los misioneros jesuitas del Caribe y el Nuevo Mundo, véase Markéta KŘÍŽOVÁ, "Las primeras impresiones del Nuevo Mundo: La isla de Española en los reportes y cartas de jesuitas centroeuropeos", in: Vida y obra de Juan Bosch en el contexto de la historia de la República Dominicana, ed. Josef Opatrný, Praga 2017, pp. 27-37.

31 Carta de Kropff, México, 29-II-1732, in: Welt-Bott, tomo. 25, pp. 74-75, no. 528. También Kropff describió varios productos tropicales, el plátano, el coco y la mandioca, así como los delfines, los caimanes y los papagayos. Ibidem, pp. 75-77. 
En los reportes de las misiones repiten alusiones a los jardines misionales. Al considerar la conveniencia del terreno para establecer una misión nueva, la calidad del suelo jugaba un papel extraordinario, y después, los misioneros dedicaban mucho tiempo a cuidarlos. Esto documenta el caso de Eusebio Francisco Kino del Segno in Trentino (su apellido original probablemente fue Chini, en alemán Kühn, y él se consideró a sí mismo como "italiano por nacimiento, alemán por educación"32). Durante el primer, fracasado intento jesuita de penetrar en California, este misionero, además de sembrar maíz, melones, sandías y calabazas, ordenó plantar "parras, granadas y membrillos; y de todo, así como de la mejor mies de las almas, nos prometemos, con el favor del cielo, mejores y copiosas cosechas" ${ }^{33}$ Esta cita, además, alude al importante esfuerzo de los misioneros jesuitas, el afán por trasplantar -en el sentido literario de la palabra- la flora europea al Nuevo Mundo, aunque ésta apenas prosperaba en el territorio árido del Noroeste de Nueva España o en las tierras bajas húmedas de la América del Sur. ${ }^{34}$ Mientras que sembraban lentejas o fundaban viñas para poder producir su propio vino de misa y así cortar expensas ${ }^{35}$, ellos participaban en el proceso global que Alfred Crosby llamó el "intercambio colombino", ${ }^{36}$ contribuyendo a la transformación del mundo conocido.

Desde el punto de vista de los misioneros, la relocación de plantas europeas familiares también podía calmar su sentido de enajenación después de abandonar definitivamente la madre patria. Además, para muchos de los jesuitas, el cultivo de las plantas y de las almas estaba estrechamente entrelazado. Como metáfora, el jardín misional encarnaba la civilización y cristiandad, opuestos a la bárbara naturaleza americana y el paganismo, lo que testificó el ya citado texto de Eusebio Francisco Kino ${ }^{37}$ Con el soporte en el Viejo tanto como el Nuevo Testamento, los jesuitas se auto-conceptualizaron como jardineros que sembraban semillas de la fe en el suelo estéril para transformarlo en un jardín edénico. El jesuita alemán Antonio Sepp intituló sus relato de la misión en Paraguay del año 1714 Paraquarischer Blumengarten [Jardín de flores paracuario] ${ }^{38}$ Pues, la creación del jardín constituía

32 Carta de Kino a la Duquesa de Aveiro, Cádiz 16-XI-1680, in: [Eusebio Francisco KINO], Kino escribe a la duquesa. Correspondencia del P. Eusebio Francisco Kino con la duquesa de Aveiro y otros documentos, ed. Ernst J. Burrus, Madrid 1964, pp. 105-115.

33 Kino a la duquesa de Aveiro, San Bruno (California), 15-XII-1683, in: Ibidem, p. 231.

34 Para el intercambio de plantas en general, véase William W. DUNMIRE, Gardens of New Spain: How Meditarrean Plants and Foods Changed America, Austin 2004.

35 Por ejemplo, Theodore TREUTLEIN, "The economic regime of the Jesuit missions in Eighteenth Century Sonora", Pacific Historical Review 8, Oakland 1939, p. 295, constó que los misioneros en Sonora, al norte de Nueva España, sin mucho éxito cultivaban vid, ya que las expensas para el vino de misa consumía gran parte del dinero destinado para el uso de la misión.

36 Alfred W. CROSBY, The Columbian Exchange: Biological and Cultural Consequences of 1492, Westport 1972.

37 Para estas metáforas, aunque estudiadas con base en la documentación de las misiones jesuitas en Canadá, véase Meridith Beck SAYRE, Cultivating Soils and Souls: The Jesuit Garden in the Americas [tesis de máster] Simon Fraser University 2007, accesible de: http://summit.sfu.ca/item/2689 [consultado el 2 de enero de 2020].

38 Anton SEPP, Jardín de flores paracuario, trad. Werner Hoffmann, Buenos Aires 1974. Para la noción jesuita del jardín (aunque más bien en el contexto europeo) véase Peter DAVIDSON, "The Jesuit Garden”, in: John O’Malley et al. (eds.), Jesuits, vol. 2, pp. 86-107; para las jardines misionales, 
un acto simbólico que sobrepasó su mero valor utilitario. Pero en la vida cotidiana valía más el uso práctico, es decir, el jardín suplementaba la dieta de los misioneros mismos, ofrecía algunos productos para vender, y también plantas medicinales, de las que se tratará más abajo. Según el misionero alemán Ignacio Pfefferkorn, "en cada misión se producía maíz, trigo, frijol, [...] azúcar de caña y chile español. [...] La producción [...] que no era utilizada en la propia misión, se destinaba a los mineros españoles." 39

Las informaciones de la correspondencia pasaron en textos impresos, destinados para documentar, propagar y alabar las dificultades y conquistas espirituales de la Compañía de Jesús en las diversas partes del mundo. En 1598, Claudio Acquaviva, el entonces general de la Orden, dio instrucciones a todos los provinciales jesuitas para que ordenaran la producción de textos historiográficos sobre sus respectivas regiones. ${ }^{40}$ Con el fin de orientar a sus lectores y para dar una idea de los retos que significaba la labor misionera en aquellos puestos lejanos, las crónicas incluían información sobre la historia y cultura de los pueblos locales y las particularidades de la naturaleza. Esto significó un gran impulso para incluir los temas del mundo natural en el corpus textual de la Compañía. ${ }^{41}$ Cada crónica jesuita se inicia con una descripción geográfica, fisiográfica y natural, y en especial de especies no conocidas en Europa, su apariencia, su uso, su importancia. ${ }^{42}$

Con respecto a las plantas, estas obras se citan hasta hoy día en como valiosas contribuciones tempranas del área de la etnobotánica. ${ }^{43}$ Por ejemplo, la Descripción geográfica, natural y curiosa de la Provincia de Sonora, cuyo autor ha sido

Michael T. BRAVO, "Mission Gardens: Natural History and Global Expansion, 1720-1820", in: Londa Schjiebinger - Claudia Swan (eds.), Colonial Botany: Science, Commerce and Politics in the Early Modern World, Philadelphia 2004, pp. 49-65.

39 Ignaz PFEFFERKORN, Sonora: Descripción de la provincia, trad. Armando Hopkins Durazo, México 2008, p. 274. La traducción al español se realizó con base en una edición en inglés (Sonora: A Description of the Province, trad. and ed. Theodore E. Treutlein, Albuquerque 1989), mientras que originalmente el texto se publicó en alemán, sobre el título Beschreibung der Landschaft Sonora..., Köln 1794-95, 2 vols.

40 John O'MALLEY, “The historiography of the Society of Jesus”, in: idem et al. (eds.), Jesuits, vol. 1, p. 7 .

41 LEDEZMA - MILLONES FIGUEROA, “Introducción”, p. 13.

42 De las obras de jesuitas españoles, la ya mencionada Historia natural y moral de Indias de José de Acosta contiene descripciones de alrededor de 150 plantas y árboles, entre ellas las que constituían los alimentos básicos indígenas, como maíz, papas, mandioca, pero también chili, cacao y coca. ACOSTA, Historia natural y moral, p. 354; lib. IV, cap. 16. Bernabé Cobo describió más de 350 plantas, a menudo bajo sus nombres nativos. Sus descripciones son más detalladas que las de Acosta. Bernabé COBO, Historia del Nuevo Mundo [1653], ed. Marcos Jiménez de la Espada, Sevilla 1892; porque el libro solamente se publicó en el siglo XIX, a pesar de las ambiciones de su autor tuvo poca influencia en el discurso jesuita. De los cronistas regionales se puede mencionar Diego de Rosales, quien en Historia general del reyno de Chile (1674) enumeró más de cien plantas medicinales. PRIETO, Missionary Scientists, p. 40. En El Orinoco ilustrado (1745) de Pedro Gumilla aparece por primera vez la descripción de la fabricación y uso del veneno vegetal curare. UDÍAS, Jesuit Contribution to Science, p. 113.

43 Para los objetivos y métodos de etnobotánica, véase Rafael LIRA - Alejandro CASAS - José BLANCAS (eds.), Mexican Ethnobotany: Interactions of People and Plants in Mesoamerica, New York 2016. Aquí, referencias a la obra de los jesuitas en pp. 3-4. 
identificado por los historiadores como Juan Nentvig, misionero oriundo de Silesia, describió los efectos beneficiosos de más de treinta plantas nativas del norte de México, enumerando otras setenta por sus nombres nativos y en castellano y constando que:

"la próvida naturaleza, o mejor diré, la providencia divina ha enriquecido a esta provincia (destituida de diestros médicos, cirujanos y boticarios) de tan excelentes producciones medicinales en hierbas, matas, raíces, gomas, frutas, minera[le] s y animales, que no se hallará tal conjunto en ninguno de los huertos botánicos de toda la Europa. Porque, además de muchísimas hierbas, etcétera, ya conocidas en la medicina [...] [ha]y otra infinitud de individuos del reino vegetable y sensitivo, que se omiten por no causar enfado". ${ }^{44}$

No todos los misioneros eran capaces de describir las plantas según estándares científicos; y algunos eran bien conscientes de este hecho. Francisco Javier Eder, un alemán quien trabajó entre los Moxos, en el noroeste de Bolivia contemporánea, advirtió en su crónica, escrita después de su destierro de la colonia: "Por lo que toca a las plantas, confieso mi ignorancia para estudiarlas. He lamentado esto y, más de una vez, cuando examinaba esta abundancia y esta diversidad de plantas, he deplorado no tener a alguien conmigo". ${ }^{45}$ Eder no sabía los nombres y tampoco aspiró a una clasificación taxonómica. Trató por lo menos de incluir informaciones prácticas, por ejemplo en torno del cultivo el cacao. ${ }^{46}$ También Ignacio Pfefferkorn se quejó de que:

"es penoso que [...] muchos [remedios] muy valiosos con los que la naturaleza ha enriquecido a Sonora, no sean más ampliamente conocidos en el mundo. Éste no es trabajo para un misionero que lejos de tener el tiempo necesario para ello, se mantiene ocupado más allá de sus fuerzas en el cuidado de los cuerpos y almas de sus indios. Deben comisionarse hombres experimentados y bien versados en esta rama del saber." ${ }^{47}$

Que los jesuitas no pasaran por alto el potencial comercial de sus jardines y el ambiente natural alrededor de sus misiones testifican, entre otros documentos, las "memorias" o listas anuales de lo que pedían para sus misiones. El importe se cubría con el "sínodo", es decir, el dinero que año tras año proporcionaba a la Compañía de Jesús la real hacienda. ${ }^{48} \mathrm{~A}$ veces, las listas contienen también menciones de los productos enviados en otra dirección, a las capitales coloniales, para ser vendidos y con las ganancias suplementar las necesidades del misionero. De las misiones

44 Juan NENTVIG, Descripción geográfica, natural y curiosa de la Provincia de Sonora [1764], ed. Germán Viveros, México 1971, p. 90. Para los aspectos botánicos de la crónica de Nentvig, véase también Luis GONZÁLEZ RODRÍGUEZ, "Religión y comercio de plantas medicinales en el noroeste colonial", in: idem, El noroeste novohispano en la época colonial, México 1993, pp. 513-543.

45 Francisco Javier EDER, Descriptio provinciae Moxitarum (1791), cit. por Jean-Pierre CLÉMENT, "El padre Eder, naturalista del país de los moxos (1750-1768), in: Kohut - Torales Pacheco (eds.), Desde los confines, p. 493.

46 Ibidem, p. 496.

47 PFEFFERKORN, Sonora, p. 67.

48 Para muestras de estas cartas, véase la edición [Eusebio Francisco KINO,] Cartas a la Procura de Misiones, ed. Manuel Ignacio Pérez Alonso, México 1987. 
del norte de Nueva España se enviaba, entre otros, la "gomilla de Sonora", resina excretada por cierto arbusto que, según el misionero alemán Ignaz Pfefferkorn, era exclusivo de Sonora.

"Hasta donde se sabe esta planta se encuentra únicamente en Sonora. [...] Todavía en 1764 no se le conocía en la ciudad de México; yo envié ahí el primer informe sobre ella y mandé también un poco de la gomilla. [...] Al siguiente año me urgieron a enviarles toda la que pudiera obtener. Cuando se toma disuelta en agua esta goma es un remedio excelente para hemorragios y sangrados." 49

El hecho de que se enviaban materiales valiosos de origen local comprueba también la orden del provincial de Nueva España, Alessandro Romano, publicada a principios del siglo XVIII, que los misioneros no envíen de las misiones cosas de valor, "ni plata, ni dinero, ni otra cosa que lo valga (exceptadas algunas medicinas) sin licencia del padre provincial". ${ }^{50}$ De las "plantas de provecho [...] que demás de dar que comer en casa, traen á su dueño dinero" ${ }^{51}$ se cultivó, entre otras, la caña de azúcar, mencionada en la crónica de José de Acosta tanto como en la de Pfefferkorn. ${ }^{52}$ El producto jesuita par excellence era la yerba mate (Ilex paraguariensis). Los habitantes nativos de Paraguay no la domesticaron, solamente coleccionaron las hojas en la selva. Después de que los jesuitas descubrieron su potencial comercial, fundaron plantaciones y, a la vez, propagaron el consumo de mate. Aunque nunca entró a los mercados europeos y la expulsión de los jesuitas terminó el proyecto comercial, eventualmente se recuperaron las plantaciones y el maté se mantuvo como bebida popular en Suramérica, como una de las herencias de la botánica económica jesuita. ${ }^{53}$

Estos sucesos económicos e intelectuales buscaron su vía en las crónicas promocionales. Además, los escolares, prominentes jesuitas que residían en Europa y se servían de los informes enviados de las regiones ultramarinas las utilizaban para formular visiones mucho más amplias, conectando el estudio de la naturaleza ultramarina con las interpretaciones teológicas, se buscaban fenómenos prodigiosos y se presentaban como señales de una revelación divina. En concordancia con la retórica barroca, los autores jesuitas plantearon que el mundo natural no era más que un repertorio de significados simbólicos. ${ }^{54}$ De este modo, la materia americana se reflejó, en especial, en las obras de Athanasio Kircher y Juan Eusebio Nieremberg, ambos de origen alemán (aunque Nieremberg nació en Madrid, de padres alemanes, y pasó allí toda su vida ${ }^{55}$ ). Aunque alabados como claras manifestaciones del alto nivel de la ciencia jesuita, los productos del encuentro virtual de estos dos sabios con el material rendido por los misioneros testifica claramente su último objetivo espiritual.

49 PFEFFERKORN, Sonora, p. 67.

50 GONZÁLEZ RODRÍGUEZ, "Religión y comercio de plantas", p. 533.

51 ACOSTA, Historia natural y moral, p. 455.

52 Ibidem, p. 456; PFEFFERKORN, p. 274.

53 Ross W. JAMIESON, "The Essence of Commodification: Caffeine Dependencies in the Early Modern World”, Journal of Social History 35/2, Oxford 2001, pp. 277-278.

54 LEDEZMA - MILLONES FIGUEROA, "Introducción", p. 17.

55 Para Nieremberg véase Hughes DIDIER, Vida y pensamiento de Juan E. Nieremberg, trad. M. Navarro Carnicer, Madrid 1976. 
Con respecto al Nuevo Mundo, la obra clave de Nieremberg era Historia Naturae, Maxima Peregrinae, publicada en una lujosa edición ilustrada en Amberes en 1635 (y nunca más en una lengua moderna). La traducción al español del título completo de la obra puede ser "historia de la naturaleza, especialmente de la exótica y sorprendente" ${ }^{56}$ Ya este título indica el énfasis de Nieremberg. Aunque el libro comienza con una justificación del estudio de la historia natural, su utilidad y provecho, la atención principal se centra no en una sistematización del conocimiento, sino en lo único, extraño, maravilloso. Lo mismo aplica para los textos en español, transcripciones de las lecturas de Nieremberg acerca de la historia natural en el Colegio Imperial de Madrid, intitulados en la edición de 1649 Curiosa y Oculta Filosofía. Primera y Segunda Parte de las Maravillas de la Naturaleza. También aquí el autor destacó los aspectos raros y exóticos de la naturaleza de lejanas tierras, especialmente del Nuevo Mundo. ${ }^{57}$ Además, es importante la postura providencial de Nieremberg: la nueva naturaleza de América le resultaba la pieza faltante que permitiría un conocimiento pleno de la obra divina. "Nunca como hoy se hizo visible la naturaleza. A los antiguos sólo se ofreció la mitad de su imagen, y así sesgadamente fue considerada. Ahora revela claramente la integridad de su rostro." ${ }^{58} \mathrm{La}$ idea de que con el conocimiento de la naturaleza americana se obtiene la imagen completa de la naturaleza implicó la percepción del Nuevo Mundo como territorio de revelación, en la que la fe superó la razón. "La naturaleza ha sido capaz de producir mayores maravillas de lo que suele comprobar la razón." 59

En lo que toca Athanasio Kircher, el polyhistor residente en Roma, su más importante -y más frecuentemente citada-contribución en el entrelazamiento entre el material rendido por el Nuevo Mundo y su análisis por el Viejo era el "museo" en el Colegio Romano, repositorio no solamente de los objetos arqueológicos romanos y etruscos, piezas de arte y máquinas curiosas, sino también de objetos e informes sobre el mundo no europeo obtenido a través de la red jesuita. Por supuesto, coleccionar productos naturales representaba un pasatiempo popular en la época y muchos de los miembros de la élite mantenían "gabinetes de curiosidades" en los que se exhibían plantas y animales o sus partes, piedras, fósiles, hallazgos arqueológicos, etc. Además de exhibir los objetos, Kircher mantuvo correspondencia con jesuitas en Brasil, Chile, Perú o México, y enviaba a ellos libros como remuneración por sus envíos de animales, plantas, curiosos e informes, pero también las

56 Domingo LEDEZMA, "Una legitimación imaginativa del nuevo Mundo: La Historia naturae, maxime peregrinae del jesuita Juan Esuebio Nieremberg”, in: Domingo Ledezma - Luis Millones FIGUEROA (eds.), El saber de los jesuitas, historias naturales y el Nuevo Mundo, Frankfurt a. M./ Madrid, 2005, p. 57.

57 MILLONES FIGUEROA, "La intelligentsia jesuita”, p. 29. Para la elaboración por Nieremberg de las fuentes jesuitas de América también UDÍAS, Jesuit Contribution, p. 114; Juan PIMENTEL, "Baroque Natures: Juan E. Nieremberg, American Wonders, and Preterimperial Natural History", in: Daniela Bleichmar et al. (eds.), Science in the Spanish and Portuguese Empires, 1500-1800, Stanford 2009, pp. 93-111.

58 Nieremberg, Historia Naturae, Maxima Peregrinae, p. 16, cit. por LEDEZMA, "Una legitimación imaginativa", p. 66.

59 Ibidem, p. 66-67. 
exquisiteces locales, como chile y chocolate ${ }^{60} \mathrm{El}$ "Museo de Kircher" se convirtió en el paradigma del espíritu científico de la Compañía así como en un modelo del afán coleccionista de la época. ${ }^{61}$ Pero al igual que Nieremberg, Kircher al fin y al cabo interpretó el Nuevo Mundo en el contexto religioso. Se esforzaba por encontrar en la naturaleza americana valores espirituales, más bien que una información inmediata para su utilización en medicina o en el intercambio comercial.$^{62}$ Reconociendo en la naturaleza americana valores y símbolos de la tradición europea, estas obras aspiraban a integrarla en la imagen tradicional del mundo y así, legitimizar la toma de posesión de ella.

\section{Farmacología, medicina, botánica científica}

Los misioneros jesuitas en las regiones ultramarinas, a diferencia de Kircher o Nieremberg, no se consideraban a sí mismos "sabios" ni "filósofos". (Pero de su parte, Nieremberg solicitó en numerosas ocasiones ser enviado al Nuevo Mundo como misionero, pero nunca se le otorgó el permiso. ${ }^{63}$ Aparentemente, para la Compañía de Jesús su valor radicaba en su alto prestigio en las cortes europeas más que en su potencial de ministro en los puestos alejados o un naturalista de práctica, hecho que de nuevo comprueba que el estudio de la naturaleza era de importancia secundaria en el proyecto jesuita.) Pero algunos de ellos, sin duda contribuyeron de modo sustancial al desarrollo y la propagación de los nuevos conocimientos. La estrategia misional de la Compañía de Jesús se basaba en una comunicación intensa, sistemática y prolongada con los nativos. La necesidad de sobrevivir en el ambiente no familiar y a veces peligroso obligaba a los misioneros a observar agudamente sus entornos. Todo esto se trasladó positivamente a sus eventuales productos científicos.

La voluntad de los jesuitas por aprender de la tradición local se manifestó muy claramente en el ámbito de la botánica, en especial de la botánica farmacéutica. Por supuesto, ninguno de los miembros de la Compañía de Jesús era médico calificado. Ignacio Loyola mantuvo una actitud reservada con respecto al estudio de la

60 Se cita a menudo su intercambio de cartas con Valentin Stansel, el misionero jesuita de origen checo, en misión en Portugal y en Brasil e interesado especialmente en la astronomía, pero también en la flora y fauna del Nuevo Mundo. Carlos ZILLER CAMENIETZKI, "The Celestial Pilgrimages of Valentin Stansel (1621-1705), Jesuit Astronomer and Missionary in Brazil”, in: Mordechai Feingold (ed.), The New Science and Jesuit Science: Seventeenth Century Perspectives, Blacksburg 2003, pp. 249-270. Para la correspondencia de Kircher en general, véase el proyecto Athanasius Kircher en la Stanford University, (accesible de: http://web.stanford.edu/group/kircher/cgi-bin/site, consultado el 2 de enero de 2020), cit. por MILLONES FIGUEROA, "La intelligentsia jesuita", p. 34.

61 Paula FINDLEN, "Scientific Spectacle in Baroque Rome: Athanasius Kircher and the Roman College Museum, in: Feingold (ed.), The New Science, pp. 225-284.

62 Esta postura, por supuesto, no era particular para la Compañía de Jesús. También otros autores, en especial los del siglo XVII, buscaban lo maravilloso, oculto y simbólico en la naturaleza americana, en vez de tratar de describirla sistemáticamente - por ejemplo, Antonio de LEÓN PINELO en su Paraíso en el Nuevo Mundo del año 1650. LEDEZMA, "Una legitimación imaginativa”, p. 54.

63 MILLONES FIGUEROA, "La intelligentsia jesuita", p. 29. 
medicina y la excluyó explícitamente de los currículos de las universidades jesuitas, a causa de considerarlo un tema ajeno a las finalidades y objetivos de la Orden. ${ }^{64}$ Pero desde los principios de la Orden se practicaba la asistencia a los enfermos, alabada por el mismo Loyola como el signo de caridad, una de las principales virtudes cristianas. Muchos de los colegios jesuitas tenían farmacias que no solamente cuidaban de los miembros de la Orden, sino también de la población local ${ }^{65}$ En el ultramar, en especial a causa de las frecuentes irrupciones de epidemias de enfermedades europeas, el trabajo como médico llegó a ser una de las tareas principales de los misioneros. En las palabras de Ignacio Pfefferkorn, "una de las más importantes responsabilidades de los misioneros consistía en la atención y vigilancia de los enfermos. [...] Para el beneficio de los enfermos yo contaba con un pequeño dispensario de varias plantas nativas y de algunas medicinas que pedía a la ciudad de México." ${ }^{\prime 6}$ Los medicamentos aparecieron muy a menudo en las memorias, a veces consumiendo la mitad del sínodo para el uso de la misión. ${ }^{67}$ Además de ser caras, las medicinas importadas muchas veces llegaban deterioradas y sin uso después del transporte prolongado. ${ }^{68}$ Por esto, los misioneros se veían obligados a surtirse de plantas y otros recursos medicinales locales.

El ya citado Juan Nentvig confirmó en su crónica que los misioneros se inspiraban en la medicina nativa. ${ }^{69}$ Pero el uso del conocimiento indígena médico y, en general, la búsqueda del punto de contacto entre los jesuitas y el mundo natural resultó problemático a causa del hecho de que existía una relación estrecha de muchas prácticas curanderas a la religión nativa y la mitología, que los jesuitas asociaban con prácticas demoníacas. Por otro lado, precisamente su auto-concepción como guardianes de la fe y los únicos propietarios de lo sobrenatural les instaba a dominar también esta área. Por ejemplo, en Chile los jesuitas se esforzaban a propósito por ganar acceso al conocimiento botánico y medicinal para poder remplazar los chamanes nativos (machis) de su posición influyente dentro de las comunidades nativas. ${ }^{70}$ Así, en las manos de los misioneros jesuitas, la botánica medicinal era no

64 Luis MARTín, La conquista intelectual del Perú: El Colegio Jesuita de San Pablo, 1568-1767, Lima 2001, p. 121.

65 PRIETO, Missionary Scientists, p. 40.

${ }_{66}$ PFEFFERKORN, Sonora, p. 279.

67 Esto consta, por ejemplo, Theodore E. TREUTLEIN, "The Jesuit Missionary in the Role of Physician", Mid-America 22/2, Chicago 1940, p. 124; y GONZÁLEZ RODRÍGUEZ, "Religión y comercio de plantas", pp, 521-522. La situación en Chile era muy similar. PRIETO, Missionary Scientists, p. 40. También en las cartas de Paraguay los misioneros se quejaban de la escasez de medicinas importadas. Eliane Cristia DENCKMANN FLECK - Robert POLETTO, "Circulation and production of knowledge and scientific practices in southern America in the 18th century: An analysis of Materia medica misionera, a manuscript by Pedro Montenegro (1710)", História, Ciências, Saúde - Manguinhos, 19/4, 2012. (Accesible de: http://www.scielo.br/pdf/hcsm/v19n4/en_02.pdf, consultado el 2 de enero de 2020, s.p.)

68 Sabine ANAGNOSTOU, "Forming, transfer and globalization of medical-pharmaceutical knowledge in South East Asian missions (17th to 18th century) - historical dimensions and modern perspectives", Journal of Ethnopharmacology 167, Oxford 2015, p. 79.

69 NENTVIG, Descripción geográfica, p. 9.

70 PRIETO, Missionary Scientists, p. 49-66. 
solamente el arma contra las enfermedades, sino también un modo para monopolizar lo sobrenatural.

Muchos conocimientos de la farmacia nativa entraron en la correspondencia de los misioneros; además, catálogos manuscritos de plantas medicinales y libros de recetas farmacéuticas circulaban entre las misiones y colegios de América. ${ }^{71}$ Y, por fin, algunos misioneros realizaron investigaciones explícitas en la materia médica americana, entre ellos Pedro Montenegro, Sigismund Asperger o Juan Steinhöffer (Esteyneffer). ${ }^{72}$ El último, oriundo de la provincia de Bohemia, no era sacerdote, sino el hermano coadjutor. Los "coadjutores temporales", o "hermanos coadjutores", estaban en el fondo de la jerarquía interna de la Orden. Se ocupaban del mantenimiento de las casas y colegios, administraban sus bienes y así como permitían a los "padres profesos" -los sacerdotes que profesaron los tres votos solemnes y el cuarto, el de la obediencia absoluta al Papa- dedicarse únicamente a los ministerios religiosos y la enseñanza. Representaban un factor indispensable en el funcionamiento de las misiones y universidades, y aunque a menudo afrontaban desprecio de parte de sus compañeros más educados, fueron precisamente ellos que, gracias a su involucramiento en la vida práctica, enriquecieron el repertorio de la ciencia jesuita. ${ }^{73}$

Esto vale precisamente para Steinhöffer, autor del compendio de botánica farmacéutica del Nuevo Mundo, Florilegio medicinal..$^{74}$ A las experiencias de Steinhöffer mismo, muchos años farmacéutico del Colegio jesuita en la Ciudad de México, se añadieron los de los misioneros en el noroeste de México en las regiones de Sonora, Sinaloa, Baja California y Tarahumara. ${ }^{75}$ La importancia de esta obra testifica no solamente el número de ediciones, sino también los testimonios de los otros jesuitas. José Neumann, también de la provincia de Bohemia, escribió sobre Steinhofer que "ayudó a los nuestros [misioneros] [...] a través del erudito libro redactado en español, [...] muy estimado por los médicos. [...] Indica los medicamentos que por ahí están al alcance y bien probados."76 También Pfefferkorn notó que el libro "era particularmente práctico porque prescribía detalladamente remedios caseros con yerbas muy conocidas". ${ }^{77}$ Aparentemente, en las misiones de Baja California

71 DENCKMANN FLECK - POLETTO, "Circulation and production of knowledge", s.p.

72 Sabine ANAGNOSTOU, "Jesuits in Spanish America: Contributions to the exploration of the American materia medica", Pharmacy in History 47/1, Madison 2005, pp. 3-17.

73 Daniel REFF, "Critical Introduction: The Historia and Jesuit Discourse", in: Andrés Pérez de Ribas, History of the Triumphs of Our Holy Faith Amongst the Most Barbarous and Fierce Peoples of the New World, trad. Daniel T. Reff, Maureen Ahern, Richard K. Danford, Tucson 1999, p. 33.

74 Juan de ESTEYNEFFER, Florilegio medicinal de todas las enfermedades..., México 1712. Después de esta primera edición, se publicó el texto en Amsterdam en 1719; otras ediciones siguieron en el siglo XVIII y también en 1888. La edición moderna era hecha por María Carmen Anzures y Bolaños, México 1978.

75 María del Carmen ANZURES Y BOLAÑOS, "El Florilegio Medicinal de Johannes Steinföffer. Una Contribución a la Etnobotánica Mexicana”, Ibero-Americana Pragensia 21, Praha 1987, p. 103; véase también Margarita Artschwage KAY, "The Florilegio Medicinal: Source of Southwest Ethnomedicine", Ethnohistory 24/3, 1977, pp. 251-259.

76 Joseph NEUMANN, Historia de las Sublevaciones Indias en la Tarahumara, trad. Simona Binková, ed. Bohumír Roedl, Praga 1994, p. 167.

77 PFEFFERKORN, Sonora, p. 280. 
era el Florilegio el libro más común después de la Biblia, los misales y los breviarios. Según las memorias, era pedido expresamente para utilizarse incluso en las misiones del río Marañón y del Alto Amazonas. ${ }^{78}$ También los inventarios hechos a raíz de la expulsión de los jesuitas en 1767 mencionan su presencia en numerosas misiones; y continuaba siendo usado por médicos en varias partes de México hasta el siglo XX. ${ }^{79}$

Muchos miembros de la Compañía por necesidad o interés se ocupaban de asuntos medicinales, esforzándose por adquirir la información necesaria. Esto documenta otro herbario farmacéutico, la Materia medica misionera de Pedro de Montenegro (1710). Montenegro se quejó que, a causa de la censura impuesta por el Santo Oficio, tuvo que esperar 18 años para poder consultar las obras de sus compañeros residentes en Brasil, Guillermo Pison y Jacobo Bonti, que escribieron un tratado sobre "varias plantas con los nombres de estas tierras". ${ }^{80}$ En su herbario Montenegro describió 150 plantas, no solamente americanas y europeas, sino también asiáticas. Y no solamente la información circulaba dentro y fuera de la Orden jesuita. El caso de la cinchona ejemplifica como los jesuitas identificaban, recogían y distribuían las especies americanas de valor, facilitaban el intercambio global de plantas y el conocimiento médico-farmacéutico. Dejando de lado la muy dudosa historia de que la condesa de Chinchón fuera curada por los jesuitas de San Pablo, no hay duda de que la cinchona o cascarilla (el nombre nativo era quina-quina), en Europa conocida en los siglos XVII y XVIII como "corteza jesuita", de veras haya sido por primera vez investigada por los farmacéuticos jesuitas del colegio San Pablo de Lima como un remedio eficaz contra las fiebres. En 1649, la Orden hizo públicas las instrucciones de cómo preparar y administrar la cinchona; y en las décadas subsecuentes mantuvo casi un monopolio en su distribución. ${ }^{81}$

Quien más se destacó entre los miembros de la Compañía de Jesús en el estudio de la flora ultramarina fue otro misionero de la provincia de Bohemia (nacido en Moravia), el hermano caodjutor Jorge José Kamel, farmacéutico del colegio de San Ignacio en Manila entre los años 1688 y 1706. A pesar de sus obligaciones cotidianas, Kamel se dedicó también activamente al estudio de la naturaleza, en los entornos de Manila durante cuatro viajes prolongados a través de las islas. Lo que es importante -y que acerca este misionero más al ámbito de la ciencia académica que a cualquiera de sus compañeros- era el hecho de que Kamel no se contentó con identificar las plantas por sus nombres locales y quizás los equivalentes españoles, sino que aspiró a establecer un sistema unificado de clasificación de plantas, en el contexto de los esfuerzos de su día, que en pocos decenios desembocaron en la

78 HAUSBERGER, "La vida cotidiana, p. 71; GONZÁLEZ RODRÍGUEZ, "Religión y comercio de plantas", p. 537; para el conocimiento de Steinhöffer en Peru véase MARTÍN, Conquista espiritual, p. 133.

79 SAYRE, Cultivating Soils and Souls, p. 22.

80 DENCKMANN FLECK - POLETTO, "Circulation and production of knowledge", s.p.

81 Juan JARAMILLO-ARANGO, "A Critical Review of the Basic Facts in the History of Cinchona", Journal of Linnaean Botany 53, Oxford 1949, pp. 272-309; Saul JARCHO, Quinine's Predecessor: Francesco Torti and the Early History of Cinchona, Baltimore 1993; PRIETO, Missionary Scientists, pp. 1-2; MARTín, Conquista espiritual, p. 125. 
taxonomía de Linneo. De hecho, Linneo mismo con gran probabilidad estudió los herbarios, dibujos y apuntes sobre la fauna de las Filipinas de Kamel, durante su estancia en Londres en 1736. (Más tarde, Linneo nombró, en honor de Kamel, la Cammelia japonica. $)^{82}$

Además, a pesar de ser un jesuita encargado de la tarea de defender y propagar la fe católica, Kamel se comunicaba extensamente con los aficionados botánicos holandeses e ingleses, entre ellos el médico de la Compañía de las Indias Orientales holandesa en Batavia y botánico entusiasta Willem Ten Rhijne o el británico Samuel Brown, residente en Madrás. Kamel incluso les enviaba sus herbarios, dibujos y notas a la Sociedad Real de Londres para el Avance de la Ciencia Natural (Royal Society of London for Improving Natural Knowledge) que desde su establecimiento en 1600 llegó a ser el centro de la investigación zoológica y botánica del mundo conocido ${ }^{83}$ La opinión de los científicos británicos sobre Kamel queda manifesta en las palabras de John Ray, presidente de la Sociedad, en su carta a Sloane de agosto de 1700: "No puedo sino ver en esto la mano de la Providencia, al instigar a tal hombre, conocedor de plantas, a dedicarse a la exploración, definición y descripción de plantas que crecen en tan lejanos rincones del mundo y describir sus virtudes y usos". ${ }^{84}$

Tales apreciaciones confirman la posición importante del jesuita bohémico entre los científicos de su tiempo. Gracias a sus contactos, los dibujos y herbarios de Kamel se preservaron hoy día mayoritariamente en Londres y parece claro que dejaron una impresión más profunda en la botánica inglesa que en la centroeuropa. Las notas que acompañaron a sus dibujos y las hojas herbarias usualmente apuntan el nombre nativo de la planta, la describen y también informan sobre su uso medicinal. Algunos textos de Kamel se publicaron ya durante su vida en los periódicos científicos ingleses, como la primera monografía sobre la flora filipina, Descriptiones fruticum et Arborum Luzonis. ${ }^{85}$ Apreciado hasta hoy día es el tratado sobre una planta que Kamel describió científicamente por primera vez, Strychnos ignatia, o el "haba de San Ignacio", en su entender casi una panacea, aunque también advirtió de sus efectos tóxicos. Es interesante que la información sobre la planta apareció también en el Florilegio medicinal de Steinhöffer, otra confirmación del constante intercambio de informaciones dentro de la red jesuita. ${ }^{86}$

82 Véase el texto clave sobre este misionero de Ondřej POKORNÝ, "La botánica a la vuelta de los siglos XVII y XVIII y Jorge José Camel, S.J.”, in: Binková - Křížová et al., Ir más allá..., pp. 218-231.

83 La Sociedad (la institución científica más antigua todavía en activo) se fundó en 1660, gracias al apoyo financiero del rey Carlos II, y en 1665 comenzó a publicar Philosophical Transactions of the Royal Society of London, el primer periódico en la historia dedicado exclusivamente a las ciencias naturales. Véase Charles Richard WELD, A History of the Royal Society with Memoirs of the Presidents, London 1848.

84 LANKESTER (ed.), Correspondence of John Ray, p. 374, op. cit. in: Pokorný, "La botánica”, p. 229.

85 "Descriptiones Fruticum et Arborum Luzonis etc.", in: [John RAY], Historia Plantarum tomus tercius: Qui est supplementum Duorum praecendentium, London 1704, Appendix I, II, pp. 43-96.

86 "An Account of the Vertues of Faba Sti Ignatii, Mentioned Last Transaction", in: Philosophical Transactions, 1699, tomo 21, p. 87 (reimpreso en: Acta eruditorum Lipsiae, 1700, tomo 10, f. 552-554; Opuscula Omnia eisdem Actis inserta, Venetia 1742, tomo 3, f. 537-538). Para las informaciones 


\section{Naturaleza del Nuevo Mundo ante la crítica ilustrada}

Con la expulsión de la Compañía de España y sus posesiones ultramarinas, comenzó en 1767 la última etapa en el desarrollo de la contemplación y presentación, por escrito, de la naturaleza americana. Muchos de los exmisioneros dejaron sus testimonios en forma de crónicas o memoriales, impresas tanto como manuscritas. Su principal objetivo era apologético, el esfuerzo por defender la Orden contra las "calumnias" de sus críticos. Por otro lado, su regreso a Europa les posibilitó sistematizar y ordenar sus experiencias, muchas veces también inspirándose en autores ilustrados y sus obras entera o parcialmente dedicadas a América, que ofrecían una imagen científica y en la mayoría de los casos despreciativa del Nuevo Mundo. Los naturalistas prominentes europeos -conde de Buffon, Cornelio de Pauw y abate Raynal, junto con el historiador William Robertson- dieron origen a la llamada "disputa del Nuevo Mundo" ${ }^{87}$ Para todos ellos, la naturaleza americana era una naturaleza hostil. En su entender, el clima y el suelo influyen sobre los seres vivos degenerándolos, debilitándolos, empequeñeciéndolos y reduciendo su fertilidad y, al fin y al cabo, privando el Nuevo Mundo de toda posibilidad de progreso en el futuro.

De los textos de estos autores, ampliamente propagados en Europa, surgió un nuevo impulso y estímulo para los jesuitas expatriados por describir e interpretar sus propias experiencias con la naturaleza americana. Más aún, porque a pesar de la obsesión de los científicos ilustrados con la observación experimental, ninguno de los autores mencionados había pisado jamás tierra americana, mientras los exmisioneros relataban desde su experiencia propia y no olvidaban acentuarlo en los prólogos de sus libros o incluso en sus títulos. Refutaban la tesis de la inferioridad natural del Nuevo Mundo y a la vez enfatizaban su conocimiento y control sobre la naturaleza y los habitantes nativos, respaldando los utillajes racionalistas de la cronología y la sistematización. Así, se acomodaban al gusto de los lectores de la época, ávidos de informaciones de primera mano, y al mismo tiempo mostraban su superioridad sobre las obras académicas de los enciclopedistas.

Es bien conocida la obra de los historiadores "criollos" que en el exilio italiano alababan las bellezas de la naturaleza y la historia de sus propios países natales. ${ }^{88}$ Pero en el debate entraron también los centroeuropeos. El ya citado alemán Ignaz Pfefferkorn quería "dar al público deseoso de países lejanos y de viajes instructivos,

sobre la "haba" véase ANAGNOSTOU, "Forming, transfer and globalization", p. 83. A principios del siglo XIX la estricnina ha sido aislada de esta planta y usada como medicamento.

${ }_{87}$ Para este debate intelectual, véase Antonello GERBI, La disputa del Nuevo Mundo. Historia de una polémica, 1750-1900, trad. de A. Alatorre, México 1982.

88 Se puede nombrar a Francisco Javier CLAVIJERO, Historia Antigua de México y de su conquista, trad. J. Joaquín de Mora, México 1844; pero también a Juan Ignacio Molina o Juan de Velasco. Véase también Karl KOHUT, "Clavijero y las disputas sobre el Nuevo Mundo en Europa y América", Destiempos 3, Ciudad de México 2008, no. 14 (= Dossier: Virreinatos, ed. Mariel Reinoso, Lillian von der Walde), pp. 52-81; Silvia Navia MÉNDEZ-BONITO, "Las historias naturales de Francisco Javier Clavijero, Juan Ignacio de Molina y Juan de Velasco", in: Domingo Ledezma - Luis Millones Figueroa (eds.), El saber de los jesuitas, historias naturales y el Nuevo Mundo, Frankfurt a. M.I Madrid, 2005, pp. 225-250 
no unas aventuras ficticias sino unas historias esenciales y útiles", basadas en sus propias experiencias. Su texto debía hacer remediar el "gran desconocimiento" que existía en Alemania sobre la provincia de Sonora, a pesar de ser ésta "una de las regiones más espléndidas en toda la América española". ${ }^{89}$ Pfefferkorn refutó explícitamente la tesis sobre la inferioridad americana al afirmar que los productos agrícolas de Sonora, como las cebollas o los rábanos, eran "mucho más grandes que en Europa". En vez de dejarse aplastar por la magnificencia amenazante de la naturaleza americana, Ignaz Pfefferkorn acentuó la "fertilidad casi increíble" de Sonora y sus potencialidades para la colonización. ${ }^{90}$

Similarmente, una fuente muy rara, el códice pictórico de Ignacio Tirsch, también elaborado después de la expulsión y hoy día guardado en la Biblioteca Nacional de Praga, ${ }^{91}$ se destaca por una concreción y minuciosidad que solamente pudo haberse basado en la propia observación. Pero, por el otro lado, se puede detectar en este testimonio (tanto como en el memorial de Pfefferkorn) una cierta idealización de la naturaleza americana. De los 46 dibujos en color de Tirsch -complementados con breves comentarios explicativos en alemán- más que la mitad de los dibujos representan la flora y fauna local, con el fin de no solamente admirar, sino describir, clasificar y buscar posibilidades para su aprovechamiento. No son propiamente dicho pinturas científicas. En las palabras de Miguel León-Portilla, en ellas "hay no poco de ingenuidad, como si se tratara de un arte popular". ${ }^{22}$ A la vez testifican la curiosidad del hombre educado en sus entornos y crean la impresión del perdido jardín edénico.

En el monasterio cisterciense austríaco de Zwettl se preservó un documento similar, producto de otro jesuita expulsado de América, Florian Paucke, y entitulado Hin und her, "Aquí y allá". Además del texto, que falta en el caso del Códice

89 Cit. y trad. al español por Manfred TIETZ, "El Padre Ignaz Pfefferkorn y su Descripción de la provincia de Sonora: de la apología religiosa a la etnografía", in: Kohut - Torales Pacheco (eds.), Desde los confines, pp. 512, 516.

90 Ibidem, pp. 48, 521-522.

91 [Ignaz TIRSCH,] Codex pictoricus mexicanus, Národní knihovna ČR [Biblioteca Nacional de la República Checa] en Praga, sección de manuscritos e impresos antiguos, sig. XVI B 18. Véase también Simona BINKOVÁ - Oldřich KAŠPAR, "La aportación de los materiales bohémicos para el estudio de la historia y cultura de América Latina (Los dibujos de Ignacio Tirsch)", Annals of the Náprstek Museum 14, Praha 1987, pp. 105-150; y la edición en inglés comentada The drawings of Ignacio Tirsch: a Jesuit missionary in Baja California, ed. Doyce B. Nunis, trad. Elsbeth Schulz-Bischof, Los Angeles 1972. Doyce Nunis opinó que por lo menos parte de estas ilustraciones fue dibujada en Baja California y el resto en México o durante el viaje de regreso a España. Pero las marcas de agua del papel utilizado, tanto como las informaciones acerca de la rigurosidad con que se confiscó a los jesuitas desterrados todo tipo de documentos, papeles y escritos, se les privó de papel y tinta y se les controlaron las demás cosas personales, llevaron a Simona Binková a confirmar que fuera de toda duda la obra había sido elaborada en Bohemia. Simona BINKOVÁ, "Las obras pictóricas de los PP. Florián Paucke e Ignacio Tirsch. Intento de una comparación", in: Manfred Tietz (ed.), Los jesuitas españoles expulsos: Su imagen y su contribución al saber sobre el mundo hispánico en la Europa del siglo XVIII (= Actas del coloquio internacional de Berlin, 7-10 de abril de 1999), Frankfurt a. M./Madrid 2001, p. 195.

92 Miguel LEÓN-PORTILLA, "Las pinturas del bohemio Ignaz Tirsch sobre México y California en el siglo XVIII”, Estudios de Historia Novohispana 5, Ciudad de México 1974, p. 92. 
pictorico mexicano de Tirsch, hay en este documento más de cien páginas de dibujos en lápiz, pluma y color. Es precisamente el gran número de dibujos en color lo que distingue la obra de Tirsch y Paucke de otras conocidas. No es que no hubieran existido ilustraciones, dibujos o grabados, que acompañaran también obras de otros autores, cartas geográficas, grabados de indígenas, o dibujos de plantas, pero lo excepcional es lo amplio y lo intencionado y sistemático del conjunto que representan las obras pictóricas de Paucke y Tirsch. ${ }^{93}$ Importante para el presente tema es el hecho de que los dos abarcaron una amplia gama de variedades de plantas (árboles y sus frutos, arbustos, hierbas y flores) y animales característicos de las respectivas regiones. En el plano más general, en ambos se reconoce el esfuerzo por dibujar una realidad diferente de la de Europa y divulgar su conocimiento en el ambiente europeo, en lengua vernacular -en este caso alemana- si bien ninguna de las dos llegó a ser publicada en su época.

No se puede comprobar con certeza cuál fue la repercusión de estos y otros testimonios de los misioneros. Pero parece claro que a pesar de su forzada salida del Nuevo Mundo y después, en 1773, de la supresión temporal de la Compañía que privó a estos autores del respaldo institucional, el acervo científico de la Orden jesuita continuaba siendo divulgado en el espacio público ilustrado. Citas de por lo menos algunos de los textos de los exmisioneros aparecieron en obras respetadas de la época ${ }^{94}$ Así, los jesuitas siguieron contribuyendo a la documentación de la naturaleza ultramarina y a su análisis.

\author{
por Markéta Kř́žzová(Praga) \\ marketa.krizova@ff.cuni.cz \\ (Escrito en español por la autora)
}

\title{
BIBLIOGRAFÍA
}

ACOSTA, José de, Historia natural y moral de las Indias [1590], Madrid: Ramón Anglés, 1894.

ANAGNOSTOU, Sabine, "Forming, transfer and globalization of medical-pharmaceutical knowledge in South East Asian missions (17th to 18th century) - historical dimensions and modern perspectives", Journal of Ethnopharmacology 167, Oxford: Elsevier, 2015, pp. 78-85.

ANAGNOSTOU, Sabine, "Jesuits in Spanish America: Contributions to the exploration of the American materia medica", Pharmacy in History 47/1, Madison: American Institute of the History of Pharmacy, 2005, pp. 3-17.

ANZURES Y BOLAÑOS, María del Carmen, "El Florilegio Medicinal de Johannes Steinföffer. Una Contribución a la Etnobotánica Mexicana”, Ibero-Americana Pragensia 21, Praga: Karolinum, 1987, pp. 103-124.

93 BINKOVÁ, "Las obras pictóricas”, pp. 192-193.

94 María Cristina TORALES PACHECO, "Los jesuitas novohispanos y la naturaleza en el siglo XVIII", in: Domingo Ledezma - Luis Millones Figueroa (eds.), El saber de los jesuitas, historias naturales y el Nuevo Mundo, Frankfurt a. M./Madrid, 2005, p. 195. En el ámbito de la geografía la estrecha relación entre la ciencia jesuita y la Ilustración probaron Simona BINKOVÁ y Carlos LAZCANO, "La herencia jesuítica y la Ilustración: el caso de la geografía y la cartografía del Noroeste de México", in: Simona Binková - Markéta Kř́ǐzová et al., Ir más allá..., pp. 103-127. 
ASÚA, Miguel de, Science in the Vanished Arcadia: Knowledge of Nature in the Jesuit Missions of Paraguay and Río de la Plata, Leiden/Boston: Brill, 2014.

BABER, Zaheer, "The Plants of Empire: Botanic Gardens, Colonial Power and Botanical Knowledge", Journal of Contemporary Asia 46/4, Abingdon: Taylor \& Francis, 2016, pp. 659-679.

BARNES, Linda L., Needles, Herbs, Gods, and Ghosts: China healing and the West to 1848, Cambridge, MA: Harvard University Press, 2005.

BARRERA-OSORIO, Antonio, Experiencing Nature: The Spanish American Empire and the Early Scientific Revolution, Austin: University of Texas Press, 2006.

BINKOVÁ, Simona, "Las obras pictóricas de los PP. Florián Paucke e Ignacio Tirsch. Intento de una comparación”, in: Manfred Tietz (ed.), Los jesuitas españoles expulsos: Su imagen y su contribución al saber sobre el mundo hispánico en la Europa del siglo XVIII (=Actas del coloquio internacional de Berlin, 7-10 de abril de 1999), Frankfurt a. M./Madrid: Vervuert/Iberoamericana, 2001, 189-206.

BINKOVÁ, Simona - LAZCANO, Carlos, "La herencia jesuítica y la Ilustración: el caso de la geografía y la cartografía del Noroeste de México", in: Simona Binková, Markéta Křížová et al., Ir más allá... (Fuentes bohemicales para el estudio comparativo de la expansión colonial española en la temprana Edad Moderna), Praga: Karolinum, 2016, pp. 103-127.

BINKOVÁ, Simona - KAŠPAR, Oldřich, "La aportación de los materiales bohémicos para el estudio de la historia y cultura de América Latina (Los dibujos de Ignacio Tirsch)", Annals of the Náprstek Museum 14, Prague: Náprstek Museum, 1987, pp. 105-150.

BINKOVÁ, Simona - KŘÍŽOVÁ, Markéta et al., Ir más allá... (Fuentes bohemicales para el estudio comparativo de la expansión colonial española en la temprana Edad Moderna), Praga: Karolinum, 2016.

BLEICHMAR, Daniela et al. (eds.), Science in the Spanish and Portuguese Empires, 1500-1800, Stanford: Stanford University Press, 2009.

BORJA GONZÁLEZ, Galaxis, "Libros americanos, autores jesuitas y público alemán: la literatura jesuítica americana en el mercado de libros del siglo XVIII", in: Karl Kohut - Ma. Cristina Torales Pacheco (eds.), Desde los confines de los imperios ibéricos: Los jesuitas de habla alemana en las misiones americanas, Frankfurt a. M./Madrid: Vervuert/Iberoamericana, 2007, pp. 663-696.

BRAVO, Michael T., "Mission Gardens: Natural History and Global Expansion, 1720-1820", in: Londa SCHIEBINGER - Claudia SWAN (eds.), Colonial Botany: Science, Commerce and Politics in the Early Modern World, Philadelphia: University of Pennsylvania Press, 2004, pp. 49-65.

BROCKWAY, Lucile H., "Science and Colonial expansion: The role of the British Royal Botanic Gardens", American Ethnologist 6/3, American Anthroplogical Association: Arlington, VA, 1979, pp. 449-465.

CAÑIZARES-ESGUERRA, Jorge, Nature, Empire and Nation: Explorations of the History of Science in the Iberian World, Stanford: Sanford University Press, 2006.

CLAVIJERO, Francisco Javier, Historia Antigua de México y de su conquista, trad. J. Joaquín de Mora, México: Porrúa, 1844.

CLÉMENT, Jean-Pierre, "El padre Eder, naturalista del país de los moxos (1750-1768), in: Karl Kohut - Ma. Cristina Torales Pacheco (eds.), Desde los confines de los imperios ibéricos: Los jesuitas de habla alemana en las misiones americanas, Frankfurt a. M./Madrid: Vervuert/Iberoamericana, 2007, pp. 481-505.

COBO, Bernabé, Historia del Nuevo Mundo [1653], ed. Marcos Jiménez de la Espada, Sevilla: Imp. de E. Rasco, 1892.

COLÓN, Cristóbal, Diario del primer y tercer viaje de Cristóbal Colón, ed. Consuelo Varela (Obras Completas de Bartolomé de Las Casas, 14 vols.), Madrid: Alianza Editorial, 1989.

COMENIUS, Johannes Amos, Opera didactica omnia, Praga: Academia, 1957.

CROSBY, Alfred W., The Columbian Exchange: Biological and Cultural Consequences of 1492, Westport: Greenwood Publishing Group, 1972.

DAVIDSON, Peter, “The Jesuit Garden”, in: John O'Malley et al (eds.), Jesuits: Cultures, Sciences, and the Arts 1540-1773, vol. 2, Toronto: University of Toronto Press, 2005, pp. 86-107.

DE VOS, Paula, "The Science of Spices: Empiricism and Economic Botany in the Early Spanish Empire", Journal of World History 17/4, Honolulu: World History Association, 2006, pp. 399-427. 
DE VOS, Paula, “An Herbal El Dorado: The Quest for Botanical Wealth in the Spanish Empire”, Endeavour 27, Oxford: Elsevier 2003, pp. 117-121, http://www.sciencedirect.com/science/article/pii/ S0160932703001091.

DENCKMANN FLECK, Eliane Cristia - POLETTO, Robert, "Circulation and production of knowledge and scientific practices in southern America in the 18th century: An analysis of Materia medica misionera, a manuscript by Pedro Montenegro (1710)“, História, Ciências, Saúde - Manguinhos 19/4, Rio de Janeiro: Casa de Oswaldo Cruz, 2012, s.p. (Accesible de: http://www.scielo.br/pdf/hcsm /v19n4/en_02.pdf, consultado el 2 de enero de 2020.)

DIDIER, Hughes, Vida y pensamiento de Juan E. Nieremberg, trad. M. Navarro Carnicer, Madrid: Fundación universitaria española, 1976.

DUNMIRE, William W., Gardens of New Spain: How Meditarrean Plants and Foods Changed America, Austin: Univeristy of Texas Press, 2004.

EASTERBY-SMITH, Sarah, Cultivating Commerce: Cultures of Botany in Britain and France, 1760-1815, Cambridge: Cambridge University Press, 2017.

ESTEYNEFFER, Juan de, Florilegio medicinal de todas las enfermedades... [1712], ed. María Carmen Anzures y Bolaños, México, D.F.: Academia Nacional de Medicina, 1978.

FEINGOLD, Mordechai (ed.), Jesuit Science and the Republic of Letters, Cambridge, Mass.: MIT Press, 2003.

FINDLEN, Paula, "Scientific Spectacle in Baroque Rome: Athanasius Kircher and the Roman College Museum", in: Mordechai Feingold (ed.), The New Science and Jesuit Science: Seventeenth Century Perspectives, Blacksburg: Springer, 2003, pp. 225-275.

FONTES DA COSTA, Palmira - LEITÃO, Henrique, "Portuguese Imperial Science 1450-1800: A Historiographical Overview“, in: Daniela Bleichmar et al. (ed.), Science in the Spanish and Portuguese Empires, 1500-1800, Stanford: Stanford University Press, 2009, pp. 35-53.

FRIDRICH, Marcus, "Archives as networks: The geography of record-keeping in the Society of Jesus (1540-1773)", Archival Science 10/3, Blacksburg: Springer, 2010, pp. 285-298.

GERBI, Antonello, La disputa del Nuevo Mundo. Historia de una polémica, 1750-1900, trad. de A. Alatorre, Ciudad de México: FCE, 1982.

GONZÁLEZ RODRÍGUEZ, Luis, "Religión y comercio de plantas medicinales en el noroeste colonial", in: Luis González Rodríguez, El noroeste novohispano en la época colonial, Ciudad de México: UNAM, 1993, pp. 513-543.

HARRIS, Steven J., "Confession-Building, Long-Distance Networks, and the Organization of Jesuit Science", Early Science and Medicine 1/3, Leiden/Boston: Brill, 1996, pp. 287-318.

HARRIS, Steven J., "Mapping Jesuit Science: The Role of Travel in the Geography of Knowledge", in: John O'Malley et al (eds.), Jesuits: Cultures, Sciences, and the Arts 1540-1773, vol. 1, Toronto: University of Toronto Press, 1999, pp. 212-240.

HARRIS, Steven J., "Jesuit Scientific Activity in the Overseas Missions, 1540-1773”, Isis 96/1, Chicago: University of Chicago Press, 2005, pp. 71-79.

HAUSBERGER, Bernd, "El padre Joseph Stöcklein o el arte de inscribir el mundo a la fe", in: Karl Kohut - Ma. Cristina Torales Pacheco (eds.), Desde los confines de los imperios ibéricos: Los jesuitas de habla alemana en las misiones americanas, Vervuert/Iberoamericana: Frankfurt a. M./Madrid 2007, pp. 631-659.

HELLYER, Marcus, "'Because the Authority of My Superiors Commands': Censorship, Physics and the German Jesuits”, Early Science and Medicine 1/3, Leiden/Boston: Brill, 1996, pp. 319-354.

JAMIESON, Ross W., "The Essence of Commodification: Caffeine Dependencies in the Early Modern World", Journal of Social History 35/2, Oxford: Oxford Academic, 2001, pp. 277-278.

JARAMILLO-ARANGO, Juan, "A Critical Review of the Basic Facts in the History of Cinchona", Journal of Linnaean Botany 53, Oxford: Linnean Society of London, 1949, pp. 272-309.

JARCHO, Saul, Quinine's Predecessor: Francesco Torti and the Early History of Cinchona, Baltimore: Johns Hopkins Univeristy Press, 1993.

[KINO, Eusebio Francisco,] Kino escribe a la duquesa. Correspondencia del P. Eusebio Francisco Kino con la duquesa de Aveiro y otros documentos, ed. Ernst J. Burrus, Madrid: Porrúa, 1964. 
[KINO, Eusebio Francisco,] Cartas a la Procura de Misiones, ed. Manuel Ignacio Pérez Alonso, México: UIA, 1987.

KOERNER, Lisbet, Linnaeus: Nature and Nation, Cambridge, MA/London: Harvard University Press, 1999.

KOHUT, Karl, “Clavijero y las disputas sobre el Nuevo Mundo en Europa y América”, Destiempos 3/14, México: Grupo Destiempos, 2008, pp. 52-81.

KOHUT, Karl - TORALES PACHECO, Ma. Cristina (eds.), Desde los confines de los imperios ibéricos: Los jesuitas de habla alemana en las misiones americanas, Frankfurt a. M./Madrid: Vervuert/ Iberoamericana, 2007.

KOSÁRY, Domokos, Culture and Society in Eighteenth-Century Hungary, trans. Zsuzsa Béres, Budapest: Corvina, 1987.

KŘÍŽOVÁ, Markéta, La ciudad ideal en el desierto: Proyectos misionales de la Compañía de Jesús y la Iglesia Morava en la América colonial, Praga: Karolinum, 2004.

KŘÍŽOVÁ, Markéta, "Las primeras impresiones del Nuevo Mundo: La isla de Española en los reportes y cartas de jesuitas centroeuropeos”, in: Josef Opatrný (ed.), Vida y obra de Juan Bosch en el contexto de la historia de la República Dominicana, Praga: Karolinum, 2017, pp. 27-37.

LEDEZMA, Domingo, "Una legitimación imaginativa del nuevo Mundo: La Historia naturae, maxime peregrinae del jesuita Juan Esuebio Nieremberg”, in: Domingo Ledezma - Luis Millones Figueroa (eds.), El saber de los jesuitas, historias naturales y el Nuevo Mundo, Frankfurt a. M./Madrid: Vervuert/Iberoamericana, 2005, pp. 53-85.

LEÓN-PORTILLA, Miguel, "Las pinturas del bohemio Ignaz Tirsch sobre México y California en el siglo XVIII", Estudios de Historia Novohispana 5, México: UNAM, 1974, pp. 89-95.

LIRA, Rafael - CASAS, Alejandro - BLANCAS, José (eds.), Mexican Ethnobotany: Interactions of People and Plants in Mesoamerica, New York: Springer, 2016.

LÓPEZ PIÑERO, José María - PARDO TOMÁS, José, La influencia de Francisco Hernández (15151587) en la constitución de la botánica y la materia médica moderna, Valencia: Cátedra e Instituto de Historia de la Medicina, 1994.

LOYOLA, Ignacio de, Obras completas (Edición manual), ed. Ignacio Iparraguirre, Cándido de Dalmases, Madrid: La Editorial católica, 1952.

MARTÍN, Luis, La conquista intelectual del Perú: El Colegio Jesuita de San Pablo, 1568-1767, Lima: Colegio de San Pablo, 2001.

MÉNDEZ-BONITO, Silvia Navia, "Las historias naturales de Francisco Javier Clavijero, Juan Ignacio de Molina y Juan de Velasco”, in: Domingo Ledezma - Luis Millones Figueroa (eds.), El saber de los jesuitas, historias naturales y el Nuevo Mundo, Frankfurt a. M./Madrid: Vervuert/Iberoamericana, 2005, pp. 225-250.

MILLONES FIGUEROA, Luis, "La intelligentsia jesuita y la naturaleza del Nuevo Mundo en el siglo XVII", in: Domingo Ledezma - Luis Millones Figueroa (eds.), El saber de los jesuitas, historias naturales y el Nuevo Mundo, Frankfurt a. M./Madrid: Vervuert/Iberoamericana, 2005, pp. $27-51$.

NAVARRO, Víctor, "Tradition and Scientific Change in Early Modern Spain: The Role of the Jesuits", in Mordechai Feingold (ed.), Jesuit Science and the Republic of Letters, Cambridge, MT: MIT Press, 2003, pp. 331-332.

NENTVIG, Juan, Descripción geográfica, natural y curiosa de la Provincia de Sonora [1764], ed. Germán Viveros, México, D.F.: AGN, 1971.

NEUMANN, Joseph, Historia de las Sublevaciones Indias en la Tarahumara, trad. Simona Binková, ed. Bohumír Roedl, Praga: Karolinum, 1994.

O’MALLEY, John, The First Jesuits, Cambridge, MA.: Harvard University Press, 1995.

O'MALLEY, John et al (eds.), Jesuits: Cultures, Sciences, and the Arts 1540-1773, Toronto: University of Toronto Press, 1999, 2005, 2 vols.

PFEFFERKORN, Ignaz, Sonora: Descripción de la provincia, trad. Armando Hopkins Durazo, México: Siglo XXI, 2008.

PIMENTEL, Juan, "Baroque Natures: Juan E. Nieremberg, American Wonders, and Preterimperial Natural History", in: Daniela Bleichmar et al. (eds.), Science in the Spanish and Portuguese Empires, 1500-1800, Stanford: Stanford University Press, 2009. 
PINO, Fermín del, "La Historia natural y moral de las Indias como género: orden y genesis literaria de la obra de Acosta", Histórica 24/2, Lima: Pontificia Universidad Católica del Perú, 2000, pp. 295-326.

POKORNÝ, Ondřej, "La botánica a la vuelta de los siglos XVII y XVIII y Jorge José Camel, S.J.", in: Simona Binková - Markéta Křížová et al., Ir más allá... (Fuentes bohemicales para el estudio comparativo de la expansión colonial española en la temprana Edad Moderna), Praga: Karolinum 2016, pp. 218-231.

PRIETO, Andrés I., Missionary Scientists: Jesuit Science in Spanish South America, 1570-1810, Nashville, Tenn.: Vanderbilt University Press, 2011.

REFF, Daniel, "Critical Introduction: The Historia and Jesuit Discourse", in: Andrés Pérez de Ribas, History of the Triumphs of Our Holy Faith Amongst the Most Barbarous and Fierce Peoples of the New World, trad. Daniel T. Reff, Maureen Ahern, Richard K. Danford, Tucson: University of Arizona Press, 1999.

SEPP, Anton, Jardín de flores paracuario, trad. Werner Hoffmann, Buenos Aires: Eudeba, 1974.

SAYRE, Meridith Beck, Cultivating Soils and Souls: The Jesuit Garden in the Americas, [tesis de máster], Simon Fraser University 2007. (Accesible de: http://summit.sfu.ca/item/2689, consultado el 2 de enero de 2020).

SCHIEBINGER, Londa, Plants and Empire: Colonial Bioprospecting in the Atlantic World, Cambridge, MA.: Harvard University Press, 2004.

SCHIEBINGER, Londa - SWAN, Claudia (eds.), Colonial Botany: Science, Commerce and Politics in the Early Modern World, Philadelphia: University of Pennsylvania Press, 2004.

STÖCKLEIN, Joseph, Allerhand so Lehr-als Geist-reiche Brief-Schrifften und Reis-beschreibungen, welche von denen Missionarios der Gesellschaft Jesu aus Beyden Indien bis anno 1731 in Europa angelangt sind, Augsburg/Gratz/Wien 1726-1761, 37 vols.

TIETZ, Manfred, "El Padre Ignaz Pfefferkorn y su Descripción de la provincia de Sonora: de la apología religiosa a la etnografía", in: Karl Kohut - Ma. Cristina Torales Pacheco (eds.), Desde los confines de los imperios ibéricos: Los jesuitas de habla alemana en las misiones americanas, Frankfurt a. M./ Madrid, Vervuert/Iberoamericana, 2007, pp. 507-540.

[TIRSCH, Ignacio,] The drawings of Ignacio Tirsch: a Jesuit missionary in Baja California, ed. Doyce B. Nunis, trad. Elsbeth Schulz-Bischof, Los Angeles: Dawson's Book Shop, 1972.

TORALES PACHECO, María Cristina, "Los jesuitas novohispanos y la naturaleza en el siglo XVIII", in: Domingo Ledezma - Luis Millones Figueroa (eds.), El saber de los jesuitas, historias naturales y el Nuevo Mundo, Frankfurt a. M./Madrid, Vervuert/Iberoamericana, 2005, pp. 195-224.

TREUTLEIN, Theodore, "The economic regime of the Jesuit missions in Eighteenth Century Sonora", Pacific Historical Review 8, Oakland: American Historical Association, 1939, pp. 289-300.

TREUTLEIN, Theodore E., "The Jesuit Missionary in the Role of Physician", Mid-America 22/2, Chicago: Loyola University, 1940, pp. 120-141.

UDÍAS, Agustín, Jesuit Contribution to Science: A History, Cham: Springer, 2015.

VAREY, Simon, et al., The Mexican Treasury: The Writings of Dr. Francisco Hernández, trad. Rafael Chabrín, Cynthia L. Chamberlein, Simon Varey, Stanford: Stanford University Press, 2001.

WADDELL, Mark, Jesuit Science and the End of Nature's Secrets, Farnham: Ashgate, 2015.

WELD, Charles Richard, A History of the Royal Society with Memoirs of the Presidents, London: John W. Parker, 1848.

ZAVADIL, Pavel, "Bohemia jesuitica in Indiis Occidentalibus. Latinská korespondence českých jezuitů z Ameriky, Filipín a Marián v českých a moravských archivech. Kritická edice” [Bohemia jesuitica in Indiis Occidentalibus. Correspondencia latina de los jesuitas bohemios de América, Filipinas y Marianas en los archivos checos y moravos], (disertación doctoral), Universidad Carolina, Praga 2011 (mecanografiada). (Accesible de https://is.cuni.cz/webapps/zzp/detail/121385/, consultado el 2 de enero de 2020).

ZILLER CAMENIETZKI, Carlos, "The Celestial Pilgrimages of Valentin Stansel (1621-1705), Jesuit Astronomer and Missionary in Brazil", in: Mordechai Feingold (ed.), The New Science and Jesuit Science: Seventeenth Century Perspectives, Blacksburg: Springer, 2003, pp. 249-270. 


\section{Breve información sobre la autora}

Markéta Kř́ižová es doctora en estudios iberoamericanos de la Universidad Carolina. Trabaja como profesora en el Centro de Estudios Ibero-Americanos de la Facultad de Filosofía, Universidad Carolina. Su investigación se centra ante todo en el problema del encuentro de Europa y las regiones ultramarinas en varias etapas históricas, la transferencia cultural, en los problemas de la "percepción del otro" y la creación de identidades colectivas. 